\title{
Predictors of time to sputum culture conversion in multi-drug-resistant tuberculosis and extensively drug-resistant tuberculosis in patients at Tshepong-Klerksdorp Hospital
}

\begin{tabular}{|c|c|}
\hline \multicolumn{2}{|c|}{$\begin{array}{l}\text { Authors: } \\
\text { Relebohile Ncha }{ }^{1} \\
\text { Ebrahim Variava }^{2,3} \\
\text { Kennedy Otwombe }^{3} \\
\text { Mary Kawonga }^{1} \\
\text { Neil A. Martinson }^{3,4}\end{array}$} \\
\hline \multicolumn{2}{|c|}{$\begin{array}{l}\text { Affiliations: } \\
\text { }{ }^{1} \text { Department of Community } \\
\text { Health, School of Public } \\
\text { Health, Faculty of Health } \\
\text { Sciences, University of } \\
\text { the Witwatersrand, } \\
\text { Johannesburg, South Africa }\end{array}$} \\
\hline \multicolumn{2}{|c|}{$\begin{array}{l}{ }^{2} \text { Department of Internal } \\
\text { Medicine, Klerksdorp- } \\
\text { Tshepong Hospital Complex } \\
\text { and School of Clinical } \\
\text { Medicine, Faculty of Health } \\
\text { Sciences, University of the } \\
\text { Witwatersrand, Johannesburg, } \\
\text { South Africa }\end{array}$} \\
\hline \multicolumn{2}{|c|}{$\begin{array}{l}{ }^{3} \text { Perinatal HIV Research } \\
\text { Unit (PHRU), SAMRC Soweto } \\
\text { Matlosana Collaborative } \\
\text { Centre for HIV/AIDS } \\
\text { and TB, University of } \\
\text { the Witwatersrand, } \\
\text { Johannesburg, South Africa }\end{array}$} \\
\hline \multicolumn{2}{|c|}{$\begin{array}{l}{ }^{4} \text { Centre of Excellence } \\
\text { in Biomedical TB } \\
\text { Research, University } \\
\text { of the Witwatersrand, } \\
\text { Johannesburg, South Africa }\end{array}$} \\
\hline \multicolumn{2}{|c|}{$\begin{array}{l}\text { Corresponding author: } \\
\text { Relebohile Ncha, } \\
\text { relebohile.ncha@gmail.com }\end{array}$} \\
\hline \multicolumn{2}{|c|}{$\begin{array}{l}\text { Dates: } \\
\text { Received: } 18 \text { Aug. } 2017 \\
\text { Accepted: } 13 \text { June } 2019 \\
\text { Published: } 26 \text { Aug. } 2019\end{array}$} \\
\hline \multicolumn{2}{|l|}{ Read online: } \\
\hline 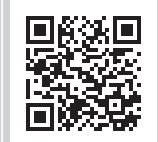 & $\begin{array}{l}\text { Scan this QR } \\
\text { code with your } \\
\text { smart phone or } \\
\text { mobile device } \\
\text { to read online. }\end{array}$ \\
\hline
\end{tabular}

Setting: Klerksdorp-Tshepong Hospital Complex MDR-TB Unit, North-West Province, South Africa.

Background: To determine the time to sputum culture conversion (TTSCC) and factors predictive of TTSCC in patients with multi-drug-resistant tuberculosis (MDR-TB) and extensively drug-resistant tuberculosis (XDR-TB) in the North-West Province.

Methods: A retrospective cohort study, abstracting patient demographic and clinical data, laboratory results, dates of sputum testing and sputum culture conversion results, from medical records of 526 MDR-TB and 47 XDR-TB patients started on TB treatment between 01 January 2012 and 31 December 2014. Predictors of TTSCC were determined by Cox proportional hazards regression.

Results: The median age was 38 years (interquartile range 31-47) with $64 \%$ being male. Overall, $79 \%$ (449) were Human Immunodeficiency Virus (HIV)-infected. The median TTSCC was 56.5 days and 162.5 days for MDR-TB and XDR-TB patients, respectively. In the multivariate analysis, age [hazard ratio (HR): $0.89,95 \%$ confidence interval (CI): 0.96-0.99], being underweight (HR: 0.631.61, 95\% CI: 0.451.03-0.882.51), Acid Fast Bacilli (AFB) positivity (HR: $0.72,95 \%$ CI: 0.51-1.01) and having XDR-TB (HR: 0.36. 95\% CI: 0.19-0.69) were predictive of longer TTSCC.

Conclusion: Predictors of TTSC allow for MDR-TB- and XDR-TB-diagnosed patients to be identified early for effective management. Those with risk factors for delayed sputum culture conversion which are being underweight and having XDR-TB should be monitored carefully during treatment so that they can achieve sputum culture conversion early.

Keywords: Nutrition; Early sputum conversion; Multi-drug-resistant TB; BMI; Predictors; Extensively drug-resistant TB.

\section{Introduction}

Multi-drug-resistant (MDR) and extensively drug-resistant (XDR) tuberculosis (TB) are both diseases of global concern. Multi-drug-resistant tuberculosis is defined as resistance to at least both isoniazid and rifampicin, while XDR-TB is defined as resistance to any fluoroquinolone and to at least one of three second-line injectable drugs (capreomycin, kanamycin and amikacin), in addition to multidrug resistance. ${ }^{1}$ According to the World Health Organization (WHO), it is estimated that there were 480000 new MDR-TB cases and 190000 deaths globally in 2014. ${ }^{2}$ Drugresistant TB (DR-TB) is associated with poor TB treatment outcomes; only $52 \%$ of MDR-TB patients and $22 \%$ of XDR-TB patients were reported cured in the 2013 cohort. $^{3}$

In managing DR-TB, sputum culture conversion from positive to negative is critical in identifying the patient's response to treatment. Sputum culture conversion is defined as two consecutive negative cultures from sputum samples obtained at least 30 days apart. Earlier sputum culture conversion during DR-TB treatment is associated with more favourable TB treatment outcomes., ${ }^{4,5}$ Differences have been observed between countries with regard to the time to sputum culture

How to cite this article: Ncha R, Variava E, Otwombe K, Kawonga M, Martinson NA. Predictors of time to sputum culture conversion in multi-drug-resistant tuberculosis and extensively drug-resistant tuberculosis in patients at Tshepong-Klerksdorp Hospital. S Afr J Infect Dis. 2019;34(1), a111. https://doi.org/10.4102/sajid.v34i1.111

Copyright: @ 2019. The Authors. Licensee: AOSIS. This work is licensed under the Creative Commons Attribution License. 
conversion (TTSCC) as well as the factors that predict TTSCC. Given the high burden of DR-TB in South Africa, it is important to determine the TTSCC and to understand the factors predictive of the TTSCC among DR-TB patients in South Africa.

According to the WHO Global Tuberculosis Report 2014, South Africa is ranked among the top 10 countries with the highest burden of DR-TB. When looking at the final treatment outcomes, South Africa has poor results. In 2010, only $72 \%$ of 7386 diagnosed DR-TB cases were started on treatment, and of these, only $15 \%$ were cured, $40 \%$ died before completing treatment and $36 \%$ were lost to follow up. ${ }^{6}$ In view of the fact that there are variations within and between provinces (geographical regions) in South Africa when it comes to the prevalence of DR-TB and treatment outcome, ${ }^{7,8}$ it is likely that there are differences between provinces in TTSCC and its predictors. It is important that we understand the TTSCC for different provinces to engage with the TB control programmes to tailor relevant interventions for different contexts.

The objective of this study was to determine the TTSCC and factors predictive of TTSCC in patients with MDR-TB and XDR-TB in the North-West Province, one of nine provinces in South Africa.

\section{Study population and methods Study design and setting}

This was a retrospective cohort study, where we abstracted clinical and demographic data from medical records of MDR-TB and XDR-TB patients who were 18 years and older and were started on MDR-TB or XDR-TB treatment from 01 January 2012 to 31 December 2014 at the KlerksdorpTshepong Hospital Complex MDR-TB Unit in Matlosana North-West Province, South Africa. The province has an estimated population of 3707000 people and has an active mining industry (gold and platinum). The province has an MDR-TB prevalence of 2.6\% (95\%CI: 1.8-3.9) and when further analysed, the MDR-TB prevalence among new TB cases is $1.9 \%$ (95\% CI: 0.8-3.1) and among previously treated cases, it is $4.3 \%$ (95\%CI: 1.4-7.1). ${ }^{8}$ When patients are diagnosed with rifampicin resistance TB on GeneXpert, they are referred to the MDR-TB Unit at Klerksdorp-Tshepong Hospital Complex for DR-TB confirmatory tests and further management once MDR/XDR-TB diagnosis is confirmed. This MDR-TB unit is one of two MDR-TB units in the North-West Province and has 76 and 20 MDR-TB and XDR-TB beds, respectively.

\section{Data collection}

Patients who were 18 years and older and were started on MDR-TB and XDR-TB treatment at the KlerksdorpTshepong MDR-TB Unit, from January 2012 to December 2014, were eligible for the study. A list of all eligible patients was obtained from the MDR-TB register on site. Medical records for all patients who met inclusion criteria were obtained and reviewed and a structured data extraction tool was used to abstract data. Explanatory variables were patient demographics, clinical characteristics and diagnostic data, TB management data, ${ }^{1}$ sputum conversion data and treatment outcomes data. The outcome variable was TTSCC, defined as the duration, in days, between DR-TB treatment initiation and the date of collection of the first sputum culture specimen that was culture negative after a prior positive culture. Data abstracted to calculate TTSCC were the date of admission (a proxy for date of MDR/XDR-TB treatment initiation) and date of the first sputum that became negative after a previous positive result. Data were captured in Redcap software version 6.17.0. ${ }^{9}$ Approval to conduct this study was obtained from the University of the Witwatersrand Human Research Ethics Committee (Medical), authorisation number: M150639, and permission to conduct the study was obtained from the Klerksdorp-Tshepong Hospital Complex.

\section{Data analysis}

Data were analysed using STATA 14 (Stata Statistical Software: Release 14, College Station, TX, USA: StataCorp LP). Continuous demographic and clinical data were described by medians and interquartile ranges (IQRs) and compared by MDR-TB and XDR-TB using the Wilcoxonsum rank test. Frequencies were determined for categorical variables and compared by MDR-TB and XDR-TB using the chi-square test. Time to sputum culture conversion was determined using the Kaplan-Meier test and the log-rank test which compared the differences between MDR-TB and XDR-TB by various explanatory variables. Predictors of TTSCC were determined by the Cox proportional hazards regression in both the univariate and multivariate analyses. Variables with $p<0.2$ in the univariate analysis as well as those defined a priori were included in the multivariate model. The final model was assessed for the proportional hazards assumption.

\section{Ethical consideration}

Ethical Clearance was issued on 26 June 2015 by the University of Witwatersrand Human Research Ethics Committee (Medical), number M150639.

\section{Results}

A total of 573 patients were enrolled in the study. The median age was 38 years (IQR 31-47), similar between MDR-TB and XDR-TB patients $(p=0.943)$. There were statistically significant differences between MDR-TB and XDR-TB patients for employment ( $43 \%$ vs $70 \%, p<0.001)$. However, there were no differences between MDR-TB and XDR-TB patients - with formal education $(89 \%$ vs $82 \%, p=0.188)$, or their smoking history ( $26 \%$ vs $21 \%, p=0.475)$, using alcohol ( $26 \%$ vs $35 \%, p=0.249$ ) or having an occupational history in gold mining ( $8 \%$ vs $13 \%, p=0.234$ ) (Table 1$)$. The median body mass index (BMI) was 18.1 (IQR: 16.2-21.2) and was similar between MDR-TB and XDR-TB patients $(p=0.521)$. Of the 565 participants tested for HIV, 448 (79\%) were

1.List of anti-TB drugs: Group 1: First line drugs: Ethambutol, Pyrazinamide; Group 2 Injectable: Kanamycin, Amikacin, Capeomycin, Viomycin; Group3: Flouroquinolones: Levofloxacin, Moxifloxacin; Group 4: second line drugs: Ethionamide, Prothionamide, Cycloserine, Terizidone, PAS; Group 5: Other. 
TABLE 1: Demographic characteristics of the participants.

\begin{tabular}{|c|c|c|c|c|c|c|c|}
\hline \multirow[t]{2}{*}{ Variables } & \multicolumn{2}{|c|}{ Overall } & \multicolumn{2}{|c|}{ MDR-TB } & \multicolumn{2}{|c|}{ XDR-TB } & \multirow[t]{2}{*}{$p$} \\
\hline & $n$ & $\%$ & $n(526)$ & $\%(92)$ & $n(47)$ & $\%(8)$ & \\
\hline \multicolumn{8}{|c|}{ Demographic characteristics } \\
\hline Age (years) median (IQR) & 38 & $31-47$ & 38 & $31-47$ & 37 & $32-47$ & - \\
\hline \multicolumn{8}{|l|}{ Race } \\
\hline Black & 568 & 99 & 522 & 99 & 46 & 98 & 0.334 \\
\hline \multicolumn{8}{|l|}{ Gender } \\
\hline \multicolumn{8}{|l|}{ Employment status } \\
\hline Employed & 261 & 46 & 228 & 43 & 33 & 70 & $<0.001$ \\
\hline \multicolumn{8}{|l|}{ Education status $\dagger$} \\
\hline Formal schooling & 474 & 88 & 437 & 89 & 37 & 82 & 0.188 \\
\hline \multicolumn{8}{|l|}{ Smoking history $\dagger$} \\
\hline Yes & 101 & 26 & 94 & 26 & 7 & 21 & 0.475 \\
\hline \multicolumn{8}{|l|}{ Alcohol history $\dagger$} \\
\hline \multicolumn{8}{|l|}{ Gold mining history } \\
\hline Yes & 47 & 8 & 41 & 8 & 6 & 13 & 0.234 \\
\hline
\end{tabular}

MDR-TB, multi-drug-resistant tuberculosis; XDR-TB, extensively drug-resistant tuberculosis; IQR, interquartile ranges.

$\dagger$, Variables have missing data.

TABLE 2: Clinical characteristics of the participants.

\begin{tabular}{|c|c|c|c|c|c|c|c|}
\hline \multirow[t]{2}{*}{ Variables } & \multicolumn{2}{|c|}{ Overall } & \multicolumn{2}{|c|}{ MDR-TB } & \multicolumn{2}{|c|}{ XDR-TB } & \multirow[t]{2}{*}{$p$} \\
\hline & $n$ & $\%$ & $n(526)$ & $\%(92)$ & $n(47)$ & $\%(8)$ & \\
\hline \multicolumn{8}{|l|}{ Clinical characteristics } \\
\hline BMI $\left(\mathrm{kg} / \mathrm{m}^{2}\right)$ median (IQR) & 18.1 & $16.2-21.2$ & 18.1 & $16.1-21.1$ & 18.1 & $16.7-21.6$ & 0.521 \\
\hline Haemoglobin (g/dL) median (IQR) & 11.1 & $9.1-13$ & 11.05 & 9-13 & 11.8 & $9.9-13.4$ & 0.134 \\
\hline Creatinine (mg/dL) median (IQR) & 67 & $55-81$ & 66 & $55-80$ & 71 & $62-87$ & 0.083 \\
\hline $\mathrm{CD} 4$ count (cells $/ \mathrm{mm}^{3}$ ) & 118 & $51-255$ & 119 & $52-255$ & 113 & $44-252$ & 0.82 \\
\hline \multicolumn{8}{|l|}{ BMI $\left(\mathrm{kg} / \mathrm{m}^{2}\right) \dagger$} \\
\hline Normal weight & 87 & 38 & 80 & 38 & 7 & 37 & \multirow{3}{*}{0.927} \\
\hline Overweight & 19 & 8 & 17 & 8 & 2 & 11 & \\
\hline Underweight & 126 & 54 & 116 & 54 & 10 & 53 & \\
\hline \multicolumn{8}{|l|}{ Past TB history } \\
\hline Yes & 345 & 60 & 314 & 60 & 31 & 66 & 0.401 \\
\hline \multicolumn{8}{|l|}{$\mathrm{X}$-ray cavities $\uparrow$} \\
\hline None & 340 & 61 & 315 & 62 & 25 & 53 & \multirow{3}{*}{0.395} \\
\hline Unilateral & 165 & 30 & 147 & 29 & 18 & 38 & \\
\hline Bilateral & 52 & 9 & 48 & 9 & 4 & 9 & \\
\hline \multicolumn{8}{|l|}{ HIV status } \\
\hline Positive & 448 & 79 & 407 & 79 & 41 & 87 & 0.161 \\
\hline \multicolumn{8}{|l|}{ ART use at admission $\dagger$} \\
\hline Defaulted & 47 & 10 & 45 & 11 & 2 & 5 & \multirow{3}{*}{0.464} \\
\hline Naïve & 142 & 32 & 128 & 32 & 14 & 34 & \\
\hline On treatment & 257 & 58 & 232 & 57 & 25 & 61 & \\
\hline \multicolumn{8}{|l|}{ CD4 count (cells $\left./ \mathrm{mm}^{3}\right) \dagger$} \\
\hline$<500$ & 402 & 93 & 369 & 94 & 4 & 11 & \multirow{2}{*}{0.268} \\
\hline$>500$ & 28 & 7 & 24 & 6 & 33 & 89 & \\
\hline \multicolumn{8}{|l|}{ Hypertension } \\
\hline Yes & 31 & 5 & 29 & 6 & 2 & 4 & 0.715 \\
\hline \multicolumn{7}{|l|}{ Diabetes } & \multirow{2}{*}{0.656} \\
\hline Yes & 8 & 1 & 7 & 1 & 1 & 2 & \\
\hline
\end{tabular}

TB, tuberculosis; MDR-TB, multi-drug resistant tuberculosis; XDR-TB, extensively drug-resistant tuberculosis; IQR, interquartile ranges; ART, antiretroviral therapy; BMI, body mass index; cluster of differentiation 4 count (CD4 count).

$\dagger$, These variables have missing data.

HIV-infected and of those with an available baseline CD4 count results on admission (430/448), 402 (93\%) had a CD4 count $<500$ cells $/ \mathrm{mm}^{3}$. Of the 448 who were HIV-positive, $257(58 \%)$ were on antiretroviral therapy (ART) on admission to hospital. There were no significant differences between MDR-TB and XDR-TB patients for their median haemoglobin (11.05 [IQR: 9-13] vs 11.8 [IQR: 9.9-13.4], $p=0.134$ ), creatinine concentration (66 [IQR: 55-80] vs 71 [IQR: 62-87], $p=0.083$ ) as well as CD4 counts (119 [IQR: 52-255] vs 113 [IQR: 44-252], $p=0.820)$, as seen in Table 2 .

Among those enrolled, 355 (62\%) had a GeneXpert MTB/ RIF (GXP) result prior to initiation of therapy of whom 352 (99\%) were positive for Mycobacterium tuberculosis with 
rifampicin resistance. Of those with a culture result on admission, 374 (87\%) had a positive sputum culture for $M$. tuberculosis. Of those enrolled, 526 (92\%) were diagnosed at baseline with MDR-TB and 47 (8\%) with XDR-TB. Of most patients, $271(38 \%)$ had treatment success, $48(8 \%)$ defaulted treatment, $137(24 \%)$ died and $171(30 \%)$ were transferred out (Table 3).
The final analysis of the TTSCC used data from 371 patients with a positive sputum culture result on admission. Of these, 329 (89\%) were MDR-TB patients, while 42 (11\%) were XDR-TB patients (Figure 1).

The overall median TTSCC for both the MDR-TB and XDRTB patients was 58.2 days (IQR 29-113); and 56 days

TABLE 3: Drug-resistant tuberculosis diagnosis and treatment outcome data of participants.

\begin{tabular}{|c|c|c|c|c|c|c|c|}
\hline \multirow[t]{2}{*}{ Variables } & \multicolumn{2}{|c|}{ Overall } & \multicolumn{2}{|c|}{ MDR-TB } & \multicolumn{2}{|c|}{ XDR-TB } & \multirow[t]{2}{*}{$p$} \\
\hline & $n$ & $\%$ & $n$ (526) & $\%(92)$ & $n(47)$ & $\%(8)$ & \\
\hline \multicolumn{8}{|l|}{ Diagnosing TB } \\
\hline \multicolumn{8}{|l|}{ Sputum smear $\dagger$} \\
\hline Negative & 206 & 41 & 193 & 42 & 13 & 29 & \multirow{2}{*}{0.186} \\
\hline Positive & 295 & 59 & 263 & 58 & 32 & 71 & \\
\hline \multicolumn{8}{|l|}{ GXP performed? $\uparrow$} \\
\hline No & 217 & 38 & 191 & 36 & 26 & 55 & \multirow{2}{*}{0.010} \\
\hline Yes & 355 & 62 & 334 & 64 & 21 & 45 & \\
\hline \multicolumn{8}{|l|}{ GXP results $\dagger$} \\
\hline Negative & 3 & 1 & 3 & 1 & 0 & 0 & \multirow{2}{*}{ - } \\
\hline Positive & 352 & 99 & 331 & 99 & 21 & 100 & \\
\hline \multicolumn{8}{|l|}{ GXP+ Rif resistant $\dagger$} \\
\hline No & 1 & 1 & 1 & 1 & 0 & 0 & \multirow{2}{*}{-} \\
\hline Yes & 351 & 99 & 330 & 99 & 21 & 100 & \\
\hline Negative & 56 & 13 & 56 & 14 & 0 & 0 & \multirow{3}{*}{ - } \\
\hline Positive & 371 & 83 & 329 & 82 & 42 & 98 & \\
\hline Inconclusive & 18 & 4 & 17 & 4 & 1 & 2 & \\
\hline \multicolumn{8}{|c|}{ DR TB treatment outcomes } \\
\hline \multicolumn{8}{|c|}{ Treatment outcome } \\
\hline Treatment success & 217 & 38 & 203 & 39 & 14 & 30 & \multirow{4}{*}{0.256} \\
\hline Deceased & 137 & 24 & 127 & 24 & 10 & 21 & \\
\hline Defaulted & 48 & 8 & 45 & 9 & 3 & 6 & \\
\hline Other & 171 & 30 & 151 & 29 & 20 & 43 & \\
\hline
\end{tabular}

TB, tuberculosis; MDR-TB, multi-drug-resistant tuberculosis; XDR-TB, extensively drug-resistant tuberculosis; DR-TB, drug-resistant tuberculosis; GXP, GeneXpert.

$\dagger$, These variables have missing data.

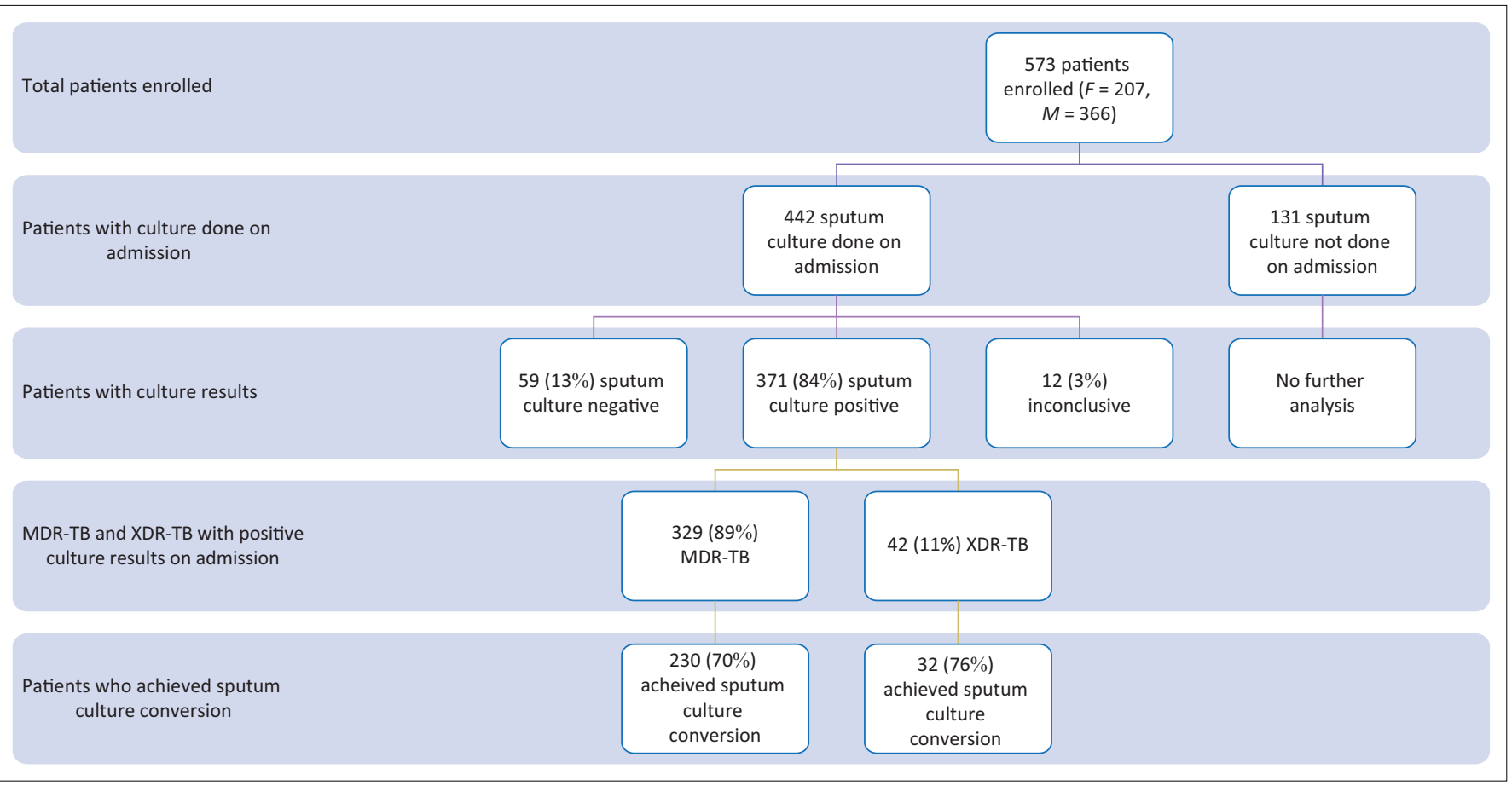

MDR-TB, multi-drug-resistant tuberculosis; XDR-TB, extensively drug-resistant tuberculosis.

FIGURE 1: Number of participants included in the analysis of time to sputum culture conversion. 
(IQR 28-99) and 139 days (IQR 54-245) for MDR-TB and XDR-TB patients, respectively (Figure 2). A total of 262/374 $(70 \%)$ achieved sputum culture conversion. Of these, 230 (69\%) were MDR-TB patients and 32 (76\%) were XDR-TB patients. Of the patients who achieved sputum culture conversion, $16(50 \%)$ XDR-TB patients were treated using 8-10 drugs and $13(41 \%)$ of the XDR-TB patients converted between 61 and 180 days, while 196 (75\%) of MDR-TB patients were treated using three to five drugs and 138 (53\%) converted in less than 60 days. Of the 230 MDR-TB patients who achieved sputum culture conversion, 111 (48\%) achieved treatment success and 35 (15\%) died; while among the 32 XDR-TB patients, 13 (41\%) achieved treatment success and $3(9 \%)$ died (Table 4$)$.

In the univariate analysis model, being underweight (HR: 0.68, 95\% CI: 0.49-0.94, $p=0.023$ ), Acid Fast Bacilli (AFB) positivity on admission (HR: $0.71,95 \% \mathrm{CI}: 0.56-0.88$, $p=0.002$ ) and having XDR-TB (HR: 0.51, 95\% CI: 0.36-0.73, $p<0.001$ ) were associated with longer TTSCC (Table 5).

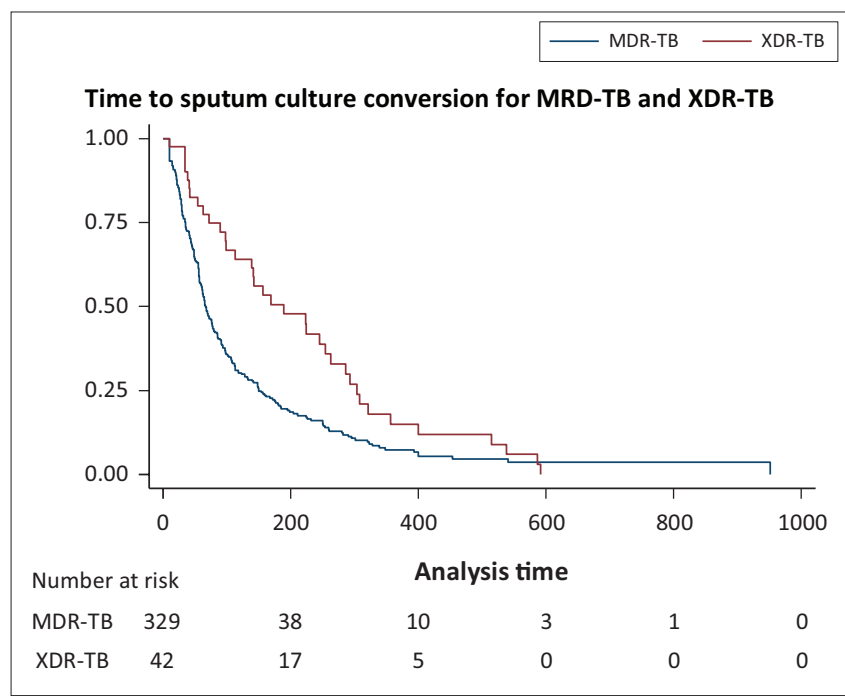

MDR-TB, multi-drug-resistant tuberculosis; XDR-TB, extensively drug-resistant tuberculosis.

FIGURE 2: Time to sputum culture conversion for multi-drug-resistant tuberculosis and extensively drug-resistant tuberculosis
In the multivariable analysis that included a priori variables, being underweight (HR: 0.63, 95\% CI: 0.45-0.88, $p=0.008$ ), having XDR-TB (HR: $0.36,95 \%$ CI: $0.19-0.69, p=0.002)$ and older age (HR: 0.98, 95\% CI: $0.96-0.99, p=0.024)$ were associated with longer TTSCC. AFB positivity on sputum on admission was marginally significant and was associated with longer TTSCC (HR: 0.72, 95\% CI: 0.51-1.01, $p=0.059$ ) (Table 5).

\section{Discussion}

Early sputum culture conversion is predictive of favourable TB treatment outcomes. ${ }^{10}$ This study reports the TTSCC and its predictors among MDR-TB and XDR-TB patients treated at Klerksdorp-Tshepong Hospital in South Africa. We found the median TTSCC for MDR-TB and XDR-TB patients to be 56 days (IQR 28-99) and 139 days (IQR 54-245), respectively. Being underweight, AFB positivity on admission and being diagnosed with XDR-TB were associated with longer TTSCC. Our study was not able to demonstrate predictors of shorter TTSCC.

Our findings regarding TTSCC were similar to those found in a KwaZulu-Natal study which reported a median TTSCC of 62 days (IQR: 48-111) for MDR-TB patients; ${ }^{11}$ but different from studies in three other provinces in South Africa (Cape Town, North West and Gauteng) which reported significantly longer (> 270 days) TTSCC for XDR-TB patients. ${ }^{12}$ These results are in keeping with what was found in Peru, where the XDR-TB patients obtained culture conversion later than MDR-TB patients. ${ }^{13}$

Evidence has shown that several factors influence both shorter and longer TTSCC. Factors that influence longer sputum conversion include the drug-resistance pattern on drug susceptibility testing (DST), a high baseline sputum bacilli count, patients with cavities on the chest X-ray and patients with a previous history of susceptible TB or MDRTB with a negative outcome such as treatment failure or relapse, while factors associated with shorter conversion include being HIV co-infected. ${ }^{14,15,16,17,18,19}$ In our study, patients who were underweight achieved longer TTSCC than those with a normal weight and this was also found in

TABLE 4: Drug-resistant tuberculosis, time to sputum culture conversion, total number of drugs and treatment outcome for patients who achieved sputum culture conversion.

\begin{tabular}{|c|c|c|c|c|c|c|c|}
\hline \multirow[t]{2}{*}{ Variables } & \multicolumn{2}{|c|}{ Overall } & \multicolumn{2}{|c|}{ MDR-TB } & \multicolumn{2}{|c|}{ XDR-TB } & \multirow[t]{2}{*}{$p$} \\
\hline & $n$ & $\%$ & $n(230)$ & $\%(88)$ & $n(32)$ & $\%(12)$ & \\
\hline \multicolumn{8}{|l|}{ TTSCC (in days) } \\
\hline$<60$ days & 138 & 53 & 129 & 56 & 9 & 28 & \\
\hline $61-180$ days & 91 & 35 & 78 & 34 & 13 & 41 & $<0.001$ \\
\hline$>180$ days & 33 & 12 & 22 & 10 & 11 & 31 & \\
\hline \multicolumn{8}{|c|}{ Total number of drugs used } \\
\hline $6-7$ drugs & 50 & 19 & 37 & 16 & 13 & 41 & - \\
\hline $8-10$ drugs & 16 & 6 & 0 & 0 & 16 & 50 & \\
\hline \multicolumn{8}{|l|}{ Treatment outcome } \\
\hline Treatment success & 124 & 47 & 111 & 48 & 13 & 41 & \\
\hline Deceased & 38 & 15 & 35 & 15 & 3 & 9 & - \\
\hline Defaulted & 13 & 5 & 12 & 5 & 1 & 3 & \\
\hline Other & 87 & 33 & 72 & 31 & 15 & 47 & \\
\hline
\end{tabular}

TTSCC, time to sputum culture conversion; MDR-TB, multi-drug-resistant tuberculosis; XDR-TB, extensively drug-resistant tuberculosis. 
TABLE 5: Univariate and multivariate analysis of predictors of time to sputum culture conversion

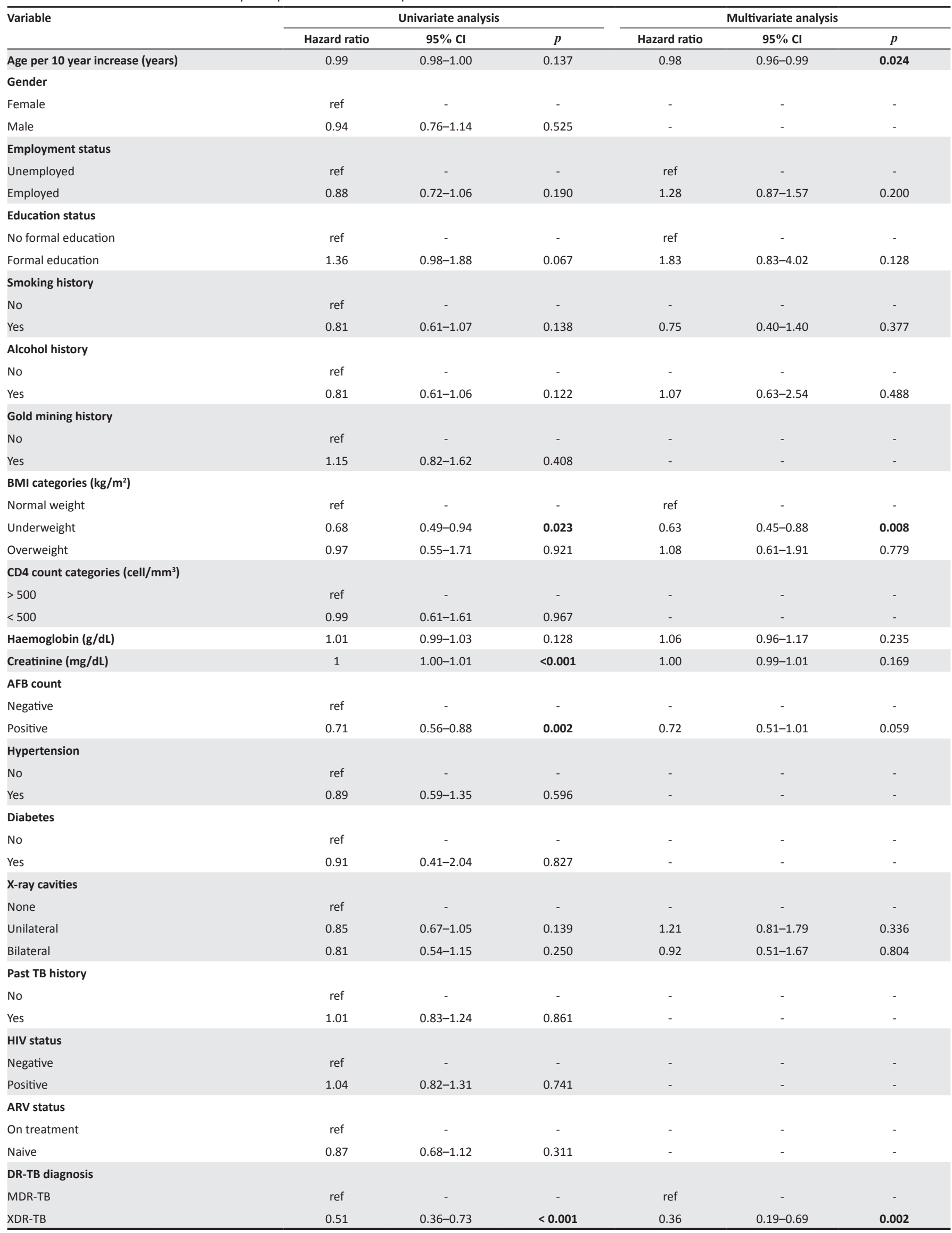

Note: The numbers in bold represent variables with $p$-values that are statistically significant.

DR-TB, drug-resistant tuberculosis; MDR-TB, multi-drug-resistant tuberculosis; XDR-TB, extensively drug-resistant tuberculosis; AFB, acid fast bacilli; ARV, antiretroviral treatment. 
the literature.$^{20}$ In our study, most patients were underweight on admission. Compared to those with normal weight, MDR-TB patients who are underweight are likely to present with clinical characteristics identified as predictors of longer TTSCC, including severe disease, cavities on the $X$-ray and a high sputum bacilli count. ${ }^{16,17,21,22}$ In the literature, the causal relationship between being underweight and DR-TB has not been proven but we do know that DR-TB can lead to wasting, increased risk of DRTB and death. ${ }^{20,23}$ Underweight patients have macro- and micronutrient deficiencies which impair immune response to infections. $^{24}$ Although the causal relationship between weight gain and shorter TTSCC has not been proven, studies have shown that being underweight can lead to unfavourable treatment outcomes such as treatment failure and death. Patients who are underweight have reduced bioavailability and malabsorption of anti-TB drugs and this can contribute to longer TTSCC and subsequently treatment failure. ${ }^{21,24}$ A randomised control trial in India showed that adding nutritional supplementation can lead to a higher sputum conversion rate. ${ }^{25}$ Therefore, patients with DR-TB who are older and underweight should be assessed for hypoalbuminemia and micronutrient deficiencies. Nutritional supplements should be provided as studies support shorter TTSCC. $.23,25,26,27$

Our findings have shown that patients with XDR-TB are likely to take longer to achieve sputum culture conversion compared to MDR-TB patients. Extensively drug-resistant TB is resistant to the most potent anti-TB drugs (rifampicin and isoniazid) as well as second-line drugs and injectables and fluoroquinolones and this makes the treatment of XDR-TB a challenge. The combination therapy for XDR-TB consists of less potent, highly toxic anti-TB drugs which are given over a longer period and this contributes to longer TTSCC. ${ }^{28}$ During our study, new drugs such as bedaquiline and delaminid were undergoing trials which have shown that the use of bedaquiline can shorten the TTSCC in treatment of DR-TB. ${ }^{29}$ Therefore, the inclusion of such drugs in the management of patients with MDR-TB and XDR-TB can lead to shorter TTSCC.

Our study confirmed that AFB positivity on the smear confirmed findings from previous studies. ${ }^{17,20,30}$ High bacilli count on admission demonstrates the high burden of resistant bacilli in the lung. Second-line drugs that are used to treat DR-TB are less efficacious, the bacilli will take longer to respond to the treatment and this leads to longer TTSCC. ${ }^{7}$ Our study demonstrated that increased age correlates with longer TTSCC. Patients develop more co-morbid diseases and have reduced immune responses and more drug-drug interactions as they age. These factors may also increase TTSCC. $^{31}$

The study has several limitations. Retrospective record reviews are limited by missing or incomplete data, and for our study, we had missing data such as the specific date of treatment initiation; therefore, we used the data of admission as a proxy for date of treatment initiation. There were missing follow-up sputum culture results as well and this influenced the number of censored patients in the survival analysis as well as the confirmation of cured patients. The impact of missing data was also evident with regard to HIV tests, CD4 count and Antiretroviral (ARV) treatment. The denominator for the CD4 count is different from the total number of HIV-positive patients and the denominator for patients on ARVs on admission is different from the number of patients who are HIV-positive and had a CD4 count.

\section{Conclusion}

The time to sputum culture conversion has both clinical and public health significance in managing DR-TB. Longer TTSCC is an indicator for poor DR-TB treatment outcomes, such as treatment failure, relapse and death. Our study has shown that age, being underweight while on DR-TB treatment, AFB positivity on admission and having XDR-TB predict longer TTSCC. Although the causal relationship between weight gain and shorter TTSCC has not been proven, studies have shown that being underweight can lead to unfavourable treatment outcomes such as death. Patients with AFB positivity should be monitored closely and their treatment optimised as they are infectious and are likely to spread the disease further.

\section{Acknowledgement}

The authors would like to acknowledge all the staff work at the PHRU at the Klerksdorp and Johannesburg office.

\section{Competing interest}

The authors have declared that no competing interest exists.

\section{Author contributions}

All authors contributed equally to this work.

\section{Funding}

This study was funded by the Perinatal HIV Research Unit SAMRC Soweto Matlosana Collaborative Centre for HIV/ AIDS and TB, University of the Witwatersrand, Johannesburg, South Africa.

\section{Data availability statement}

Data sharing is not applicable to this article as no new data were created or analysed in this study.

\section{Disclaimer}

The views and opinions expressed in this article are those of the authors and do not necessarily reflect the official policy or position of any affiliated agency of the authors. 


\section{References}

1. World Health Organization. Definitions and reporting framework for tuberculosis2013 revision. Geneva: WHO; 2013.

2. World Health Organization. Global tuberculosis report 2015. Geneva: WHO; 2015

3. World Health Organization. Global tuberculosis report 2016. Geneva: WHO; 2016

4. O'Donnell MR. Treatment outcomes for extensively drug-resistant tuberculosis and HIV co-infection. Emerg Infect Dis J. 2013;19(3):416-424. https://doi.org/ 10.3201/eid1903.120998

5. Kurbatova EV, Cegielski JP, Lienhardt C, et al. Sputum culture conversion as a prognostic marker for end-of-treatment outcome in patients with multidrugresistant tuberculosis: A secondary analysis of data from two observational cohor studies. Lancet Resp Med. 2015;3(3):201-209. https://doi.org/10.1016/S2213 2600(15)00036-3

6. World Health Organization. Global tuberculosis report 2014. Geneva: WHO; 2014

7. Department of Health. Management of drug resistant TB: Policy guidelines. Pretoria: Department of Health, Government of South Africa; 2013.

8. National Institute for Communicable Diseases. South African tuberculosis drug resistant survey 2012-2014. Gauteng, South Africa: National Institute of Communicable diseases, Sandringham; 2016.

9. Harris PA, Taylor R, Thielke R, Payne J, Gonzalez N, Conde JG. Research electronic data capture (REDCap) - A metadata-driven methodology and workflow process for providing translational research informatics support. I Biomed Inform for providing translational research informatics support. J

10. Feng-zeng Z, Levy M, Sumin W. Sputum microscopy results at two and three months predict outcome of tuberculosis treatment [Notes from the Field]. Int J Tuberc Lung Dis. 1997;1(6):570-572.

11. Heller T, Lessells $R$, Wallrauch $C$, et al. Community-based treatment for multidrugresistant tuberculosis in rural KwaZulu-Natal, South Africa. Int J Tuberc Lung Dis. 2010;14(4):420-426.

12. Pietersen $E$, Ignatius $E$, Streicher EM, et al. Long-term outcomes of patients with extensively drug-resistant tuberculosis in South Africa: A cohort study. Lancet. 2014;383(9924):1230-1239. https://doi.org/10.1016/S0140-6736(13)62675-6

13. Mitnick CD, Shin SS, Seung KJ, et al. Comprehensive treatment of extensively drug-resistant tuberculosis. N Eng J Med. 2008;359(6):563-574. https://doi. org/10.1056/NEJMoa0800106

14. Holtz T, Lancaster J, Laserson K, Wells C, Thorpe L, Weyer K. Risk factors associated with default from multidrug-resistant tuberculosis treatment, South Africa, 1999-2001. Int J Tuberc Lung Dis. 2006;10(6):649-655.

15. Qazi F, Khan U, Khowaja $S$, et al. Predictors of delayed culture conversion in patients treated for multidrug-resistant tuberculosis in Pakistan. Int J Tuberc Lung Dis. 2011;15(11):1556-1560. https://doi.org/10.5588/ijtld.10.0679

16. Kurbatova E, Gammino V, Bayona J, et al. Predictors of sputum culture conversion among patients treated for multidrug-resistant tuberculosis. Int J Tuberc Lung Dis. 2012;16(10):1335-1343. https://doi.org/10.5588/ijtld.11.0811
17. Brust JC, Lygizos M, Chaiyachati K, et al. Culture conversion among HIV co-infected multidrug-resistant tuberculosis patients in Tugela Ferry, South Africa. PLoS One. 2011;6(1):e15841. https://doi.org/10.1371/journal.pone.0015841

18. Telzak EE, Fazal BA, Pollard CL, Turett GS, Justman JE, Blum S. Factors influencing time to sputum conversion among patients with smear-positive pulmonary tuberculosis. Clin Infect Dis. 1997;25(3):666-670. https://doi.org/10.1016/j.ijid. 2015.11.018

19. Kim J, Kwak N, Lee $\mathrm{HY}$, et al. Effect of drug resistance on negative conversion of sputum culture in patients with pulmonary tuberculosis. Int J Infect Dis. 2016;42:64-68. https://doi.org/10.1016/j.ijid.2015.11.018

20. Putri FA, Burhan $E$, Nawas $A$, et al. Body mass index predictive of sputum culture conversion among MDR-TB patients in Indonesia. Int J Tuberc Lung Dis. 2014;18(5):564-570. https://doi.org/10.5588/ijtld.13.0602

21. Podewils L, Holtz $T$, Riekstina $V$, et al. Impact of malnutrition on clinical presentation, clinical course, and mortality in MDR-TB patients. Epidemiol Infec. 2011;139(01):113-120. https://doi.org/10.1017/S0950268810000907

22. Holtz TH, Sternberg $M$, Kammerer $S$, et al. Time to sputum culture conversion in multidrug-resistant tuberculosis: Predictors and relationship to treatment outcome. Ann Intern Med. 2006;144(9):650-659. https://doi.org/10.7326/0003 4819-144-9-200605020-00008

23. Bhargava A, Chatterjee $M$, Jain $Y$, et al. Nutritional status of adult patients with pulmonary tuberculosis in rural central India and its association with mortality PLoS One. 2013;8(10):e77979. https://doi.org/10.1371/journal.pone.0077979

24. Padmapriyadarsini C, Shobana M, Lakshmi M, Beena T, Swaminathan S. Undernutrition \& tuberculosis in India: Situation analysis \& the way forward. Ind J Med Res. 2016;144(1):11-20. https://doi.org/10.4103/0971-5916.193278

25. Jahnavi $\mathrm{G}$, Sudha $\mathrm{CH}$. Randomised controlled trial of food supplements in patients with newly diagnosed tuberculosis and wasting. Singapore Med J. 2010;51(12) 957-962.

26. Paton NI, Chua Y-K, Earnest A, Chee CBE. Randomized controlled trial of nutritiona supplementation in patients with newly diagnosed tuberculosis and wasting. Am J Clin Nutr. 2004;80(2):460-465. https://doi.org/10.1093/ajcn/80.2.460

27. Seung KJ, Omatayo DB, Keshavjee S, Furin JJ, Farmer PE, Satti H. Early outcomes of MDR-TB treatment in a high HIV-prevalence setting in Southern Africa. PLoS One. 2009;4(9):e7186. https://doi.org/10.1371/journal.pone.0007186

28. Caminero JA, Sotgiu G, Zumla A, Migliori GB. Best drug treatment for multidrugresistant and extensively drug-resistant tuberculosis. Lancet Infect Dis. 2010;10(9):621-629. https://doi.org/10.1016/S1473-3099(10)70139-0

29. Diacon AH, Pym A, Grobusch MP, et al. Multidrug-resistant tuberculosis and culture conversion with bedaquiline. N Engl J Med. 2014;371(8):723-732. https:// doi.org/10.1056/NEJMoa1313865

30. Güler M, Ünsal E, Dursun B, Aydln Ö, Capan N. Factors influencing sputum smear and culture conversion time among patients with new case pulmonary tuberculosis. Int J Clin Pract. 2007;61(2):231-235. https://doi.org/10.1111/ j.1742-1241.2006.01131.x

31. Hoheisel G, Hagert-Winkler A, Winkler J, et al. Pulmonary and pleural tuberculosis in the elderly. Med Klin (Munich) 2009;104(10):772-779. https://doi.org/10.1007/ s00063-009-1163-y 